\title{
Analysis of the potential inappropriate use of medications in pediatric outpatients in China
}

\author{
Cui Jing, Zhao Lei, Liu Xianghong, Liu Mengyujie and Zhong Lihong*
}

\begin{abstract}
Background: The appropriate use of medications is essential in children. Yet, detailed information on how drugs are being prescribed and dispensed to pediatric populations is not documented in China.

Aim: The study objective was to analyze the details of medicine use and categorize the types of inappropriate use of medications on children.

Methods: A retrospective cross-sectional study was conducted on the prescriptions of pediatric outpatients aged $<18$ years from 2019 to 2020 at a major Chinese tertiary academic center. Each age group's demographic and clinical characteristics were collected, and the ratios of inappropriate prescriptions were analyzed.

Results: The total number of pediatric outpatients was 652,152, and $49.37 \%$ (322000) were prescribed medications, in which the most widely used medicines were respiratory, anti-infectives, and Traditional Chinese Medicines (TCMs). The prevalence rate of inappropriate prescriptions reached 20.49\%, and in 2019 it was higher (21.71\%) than that in 2020 (18.36\%). The top three common inappropriate categories were indication-related off-label drug use, improper administration frequency, and overdosing, accounting for $67.93,17.80$ and $11.06 \%$ of all inappropriate prescriptions, respectively. The inappropriate prescriptions were more likely seen in patients aged $2-5$ years and respiratory medicines.

Conclusions: The study findings indicate that inappropriate drug use in pediatric outpatients is still common, and great attention needs to be paid. More prospective trials are required to identify the effectiveness, safety, and necessity of off-label drug use of medicines in children.
\end{abstract}

Keywords: Prescription, Pediatric, Outpatient, Drug utilization, Inappropriate medicine use

\section{Introduction}

Data from the sixth national census shows that the population in the age range of $0-14$ years reached 253 million, accounting for $17.95 \%$ of the total population in 2020 in China [1]. The number of children who get sick each year accounts for about $20 \%$ of the total number of patients [2]. The children's unique physiological characteristics result in significantly different absorption, distribution, metabolism, and excretion of drugs compared

\footnotetext{
*Correspondence: lihongtougao@163.com

Department of Pharmacy, Qilu Hospital of Shandong University, Jinan, China
}

to adults [3]. The potential of injury is higher in young children and infants. It can result in severe morbidity and even mortality, so the choice and use of drugs in children should be paid more attention [4]. However, unlicensed and off-label prescriptions for children are common in many countries [5-7]. Monitoring the safety of medicine use in children is very important, especially the use of medicines outside the specifications described in the license (e.g., in terms of formulation, indications, contraindications, or age) constitutes off-label and off-license use, and these are a significant area of concern [8].

In current studies, the information on medicine use was mostly collected from the national pharmaceutical 
claims database or nationwide health and Welfare's registries, but these databases did not provide the rationality of drug use [9-11]. The details of how drugs are being prescribed and dispensed to pediatric populations are not available for the Chinese population. Understanding this information can identify areas of process improvement and can enhance the patient safety profile.

\section{Method}

A retrospective cross-sectional study was conducted on a major Chinese tertiary academic center to analyze the details of medicine use and categorize the types of inappropriate use of medications in children. Ethical approval was granted by the Medical Ethics Committee of Qilu Hospital of Shandong University (Approval number KYLL-202011-136).

\section{Data sources}

The prescriptions of pediatric outpatients aged $<18$ years prescribed during January 1, 2019 and December 31, 2020 were extracted from the Hospital Information System (HIS) and Clinical rational drug use monitoring system. If the patient did not purchase the medicine prescribed by the doctor from the hospital, the medication records were missing in HIS, thus, this part of the data was excluded.

Patients were grouped into one of four age groups $(<2$, $2-5,6-11$, and $12-17$ years) $[9,12-15]$, and the following information on patient's prescription was collected: age, sex, insurance type, medication name, and dosage, administration route and spending of drugs (converted from Chinese Yuan to US\$ based on the exchange rate of 6.90 Yuan per US\$, which was the average exchange rate for 2019 and 2020). Medications were classified based on the Anatomical Therapeutic Chemical (ATC 2021) classification proposed by the World Health Organization (WHO) [16]. The medications not registered in ATC were classified based on their clinical applications.

\section{Inappropriate prescription categories}

The rationality of prescriptions was analyzed by the Clinical rational drug use monitoring system, which was developed by the author's hospital to monitor the inappropriate use of drugs. The definition and classification of inappropriate prescriptions in this system refer to the Hospital Prescription Review Management Specification (Trial) issued by the National Health Commission of the People's Republic of China, and it can automatically review, analyze and summarize the rationality of prescription drug use [13].

Inappropriate prescriptions were categorized into the following nine categories: (1) overdosing, (2) improper administration frequency, (3) improper administration routes, (4) contraindication, (5) improper diluent volume, (6) medications not allowed for pediatric patients, (7) improper drug combination, (8) repeated administration, and (9) indication-related off-label drug use.

\section{Statistical analysis}

All analyses were conducted using SPSS Statistics 25.0 (IBM, Armonk, N Y). A descriptive analysis was conducted on the patient's demographic, clinical, and medication characteristics. Mean (SD) was computed for continuous data. Frequencies and percentages were calculated for categorical variables. The prevalence and inappropriate medicine use changes were also analyzed for all the children between 2019 and 2020. The Chisquare test was used to compare the differences in the prevalence rates, and the T-test was used to determine the significance of differences between means.

\section{Results}

The total number of pediatric outpatients was 652,152 , of which $59.09 \%$ were boys, and $49.37 \%$ (322000) were prescribed medications. Among patients who received medications, $27.04 \%$ received injected formulations, and basic medical insurance covered $1.13 \%$. The total spending on drugs was US $\$ 9.86$ million, an average of 2.40 kinds of medications and US $\$ 30.61$ per patient per visit. The prescription details of demographic and drug character were showed in Table 1.

The proportion of children who received medications (RR $0.814 ; 95 \%$ CI $0.810-0.819 ; p<0.001$ ), the average number of medications prescribed $(p<0.001)$, and the proportion of children who received injected formulations (RR 0.773; 95\%CI 0.765-0.783; $p<0.001$ ) decreased significantly in 2020 compared with 2019 . The spending of drugs on one child in each visit increased with age and was higher in 2020 than that in $2019(p<0.001)$.

\section{Drug utilization}

The most widely used medicines were respiratory, antiinfectives, traditional Chinese medicines (TCMs), drugs related to the nervous system, alimentary tract, and dermatological drugs (Fig. 1). Antibiotics accounted for 92.13\% $(97,925 / 106425)$ of all the anti-infectives. However, the prevalence rates for respiratory, TCMs, and anti-infectives were higher for children aged $<6$ years than those aged $>6$ years. However, the prevalence rates were lower for drugs related to the nervous system, alimentary tract, and dermatological medications for children aged $<6$ years.

Table 2 lists all the medicines whose prevalence $>20$ users per 1000 person-years in any age group (except for the solvent). As shown in Table 2, the top 3 medications for respiratory were ambroxol hydrochloride, 


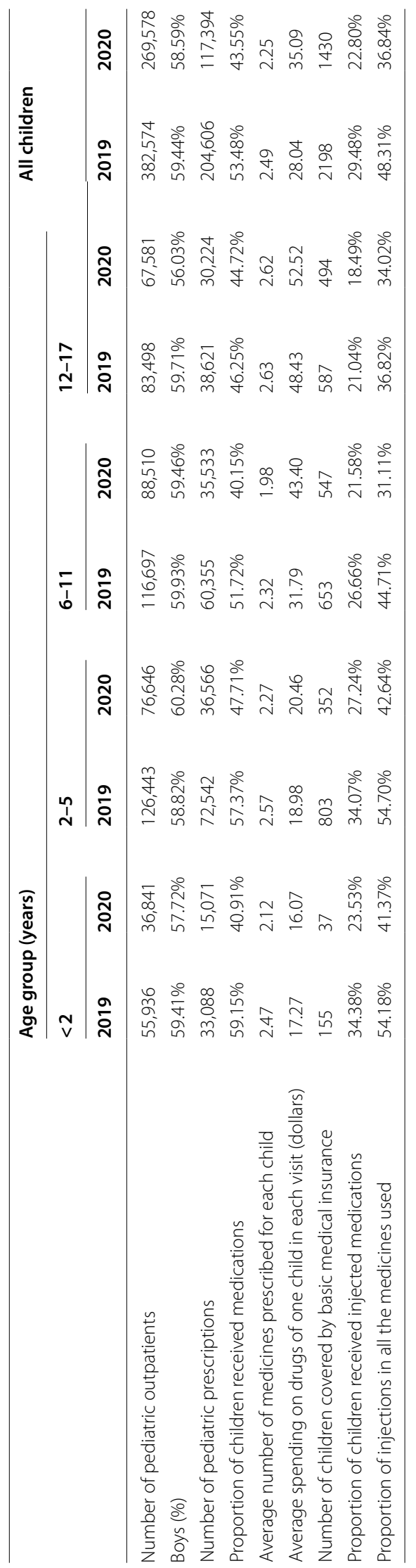




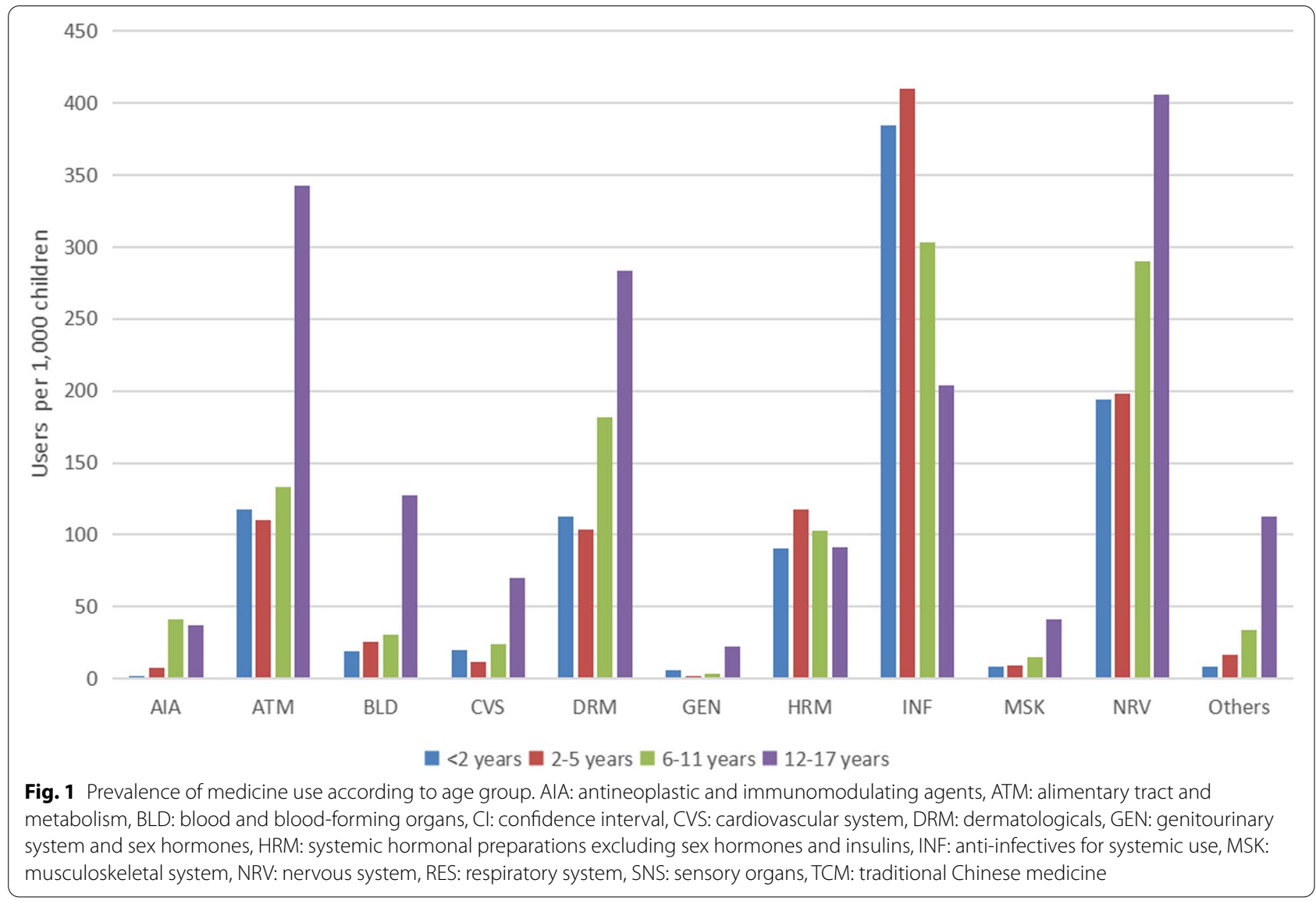

budesonide, and terbutaline. However, the medicine most widely used in children aged $<2$ years was ying'er zhike mixture, a hospital preparation used to treat cough. The top 3 anti-infectives were ceftriaxone, cefixime, and cefaclor. The top TCMs were dehydroandrographolide succinate, xiao'er jinqiao granule, and feilike mixture.

Table 2 also shows the changes in the prevalence of medicine use for all children between 2020 and 2019 . There were significant decreases in the use of respiratory, anti-infectives, TCMs, and systemic hormones. The prevalence rate of dehydroandrographolide succinate, ambroxol, and dexamethasone, the top 3 medications most widely used in 2019 , dropped about $50 \%$, and the total prevalence rate of the major antibiotics dropped nearly $25 \%$. This contrasted with a significant increase in the drugs related to the nervous system and dermatological medicines.

\section{Inappropriate types of prescriptions}

Of the 322,000 prescriptions analyzed, 65,974 were considered inappropriate, reaching a prevalence rate of $20.49 \%$. Among these inappropriate prescriptions, a total of 73,358 inappropriate cases were found, and 6373 prescriptions had more than one case. As shown in Table 3, the top 3 common inappropriate categories included indication-related off-label drug use(67.93\%), improper administration frequency (17.80\%), and overdosing (11.06\%).

As shown in Table 4, inappropriate prescriptions were more in 2019 than in 2020, and the rate was higher $(21.71 \%, 44,421 / 204606)$ than in 2020 (18.36\%, 21,553/117394) (RR 0.846; 95\%CI 0.833-0.858; $<<0.001$ ). In age groups, overdosing, improper administration frequency, improper administration routes, improper diluent volume, and indication-related off-label drug use were more likely seen in patients aged $2-5$ years. While contraindications, medicines not allowed for pediatric patients, improper drug combination, and repeated administration were more likely seen in patients aged $>12$ years.

Among all the inappropriate drug use, respiratory medicines accounted for $40.15 \%$, followed by anti-infective drugs (14.33\%) and TCMs (10.61\%). Contraindication was more likely to appear in the alimentary tract and metabolism drugs. Medication not allowed for pediatric patients was more likely to appear in nervous system drugs, and improper drug combinations were more likely to occur in antineoplastic and immunomodulating drugs. 


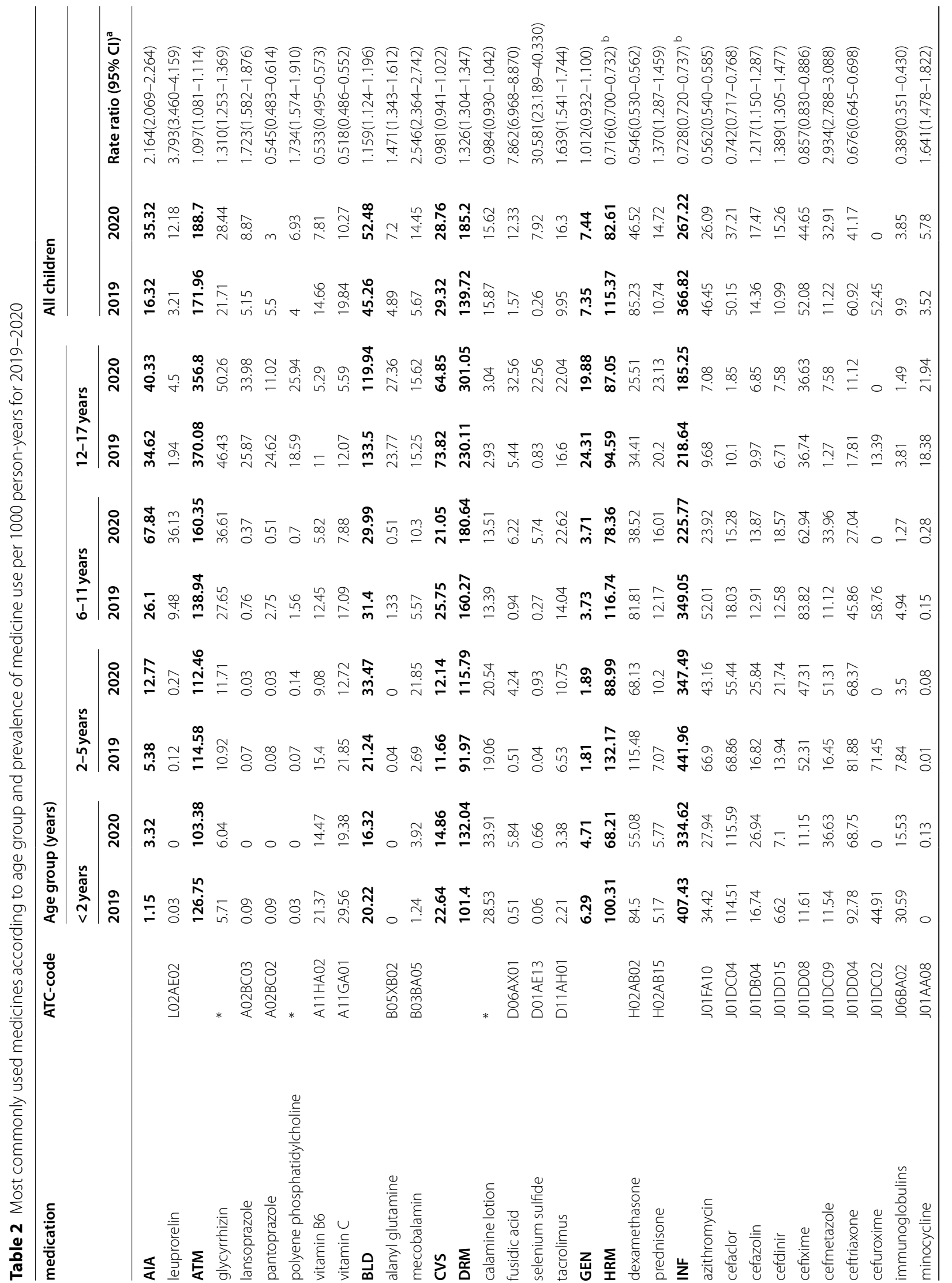




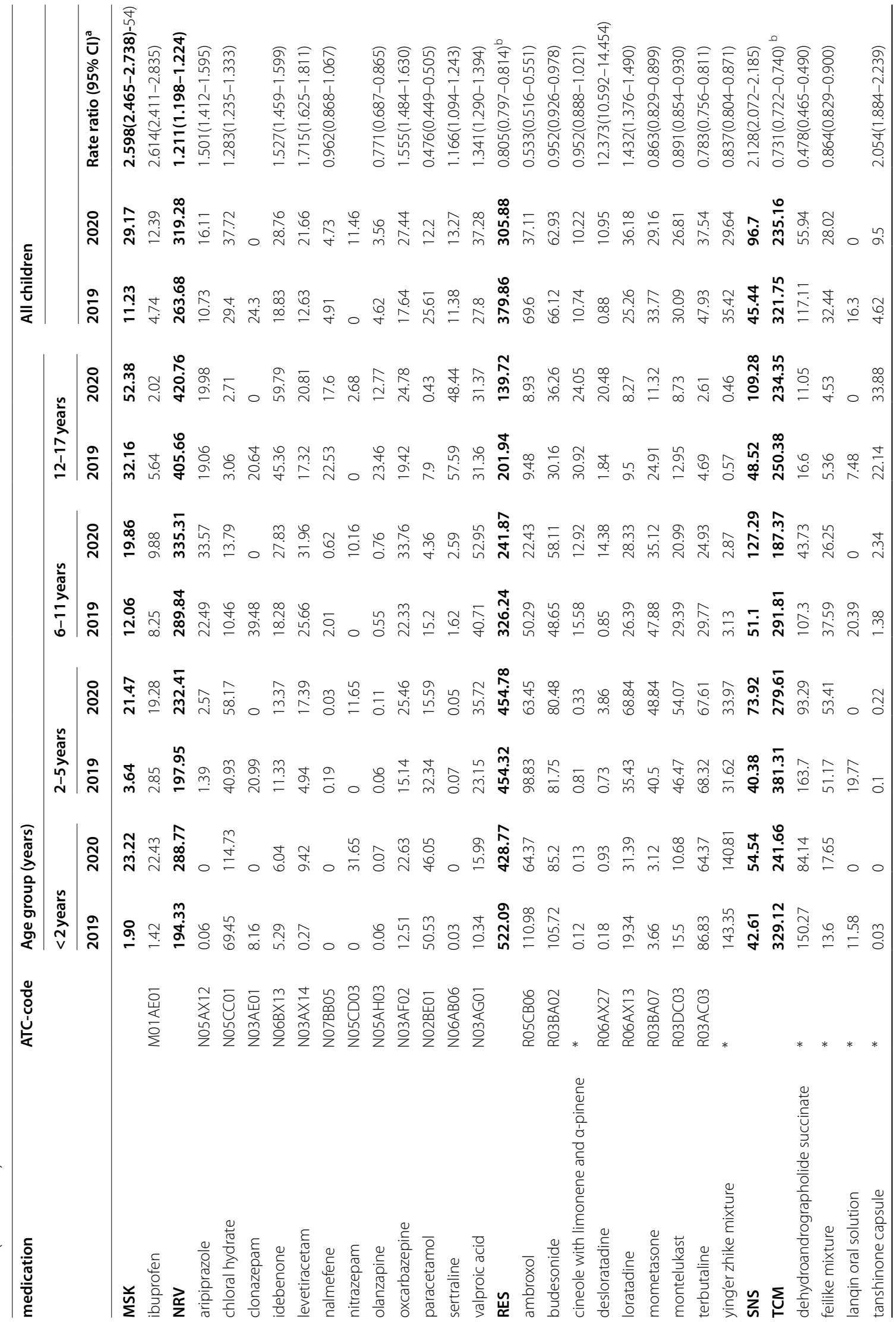




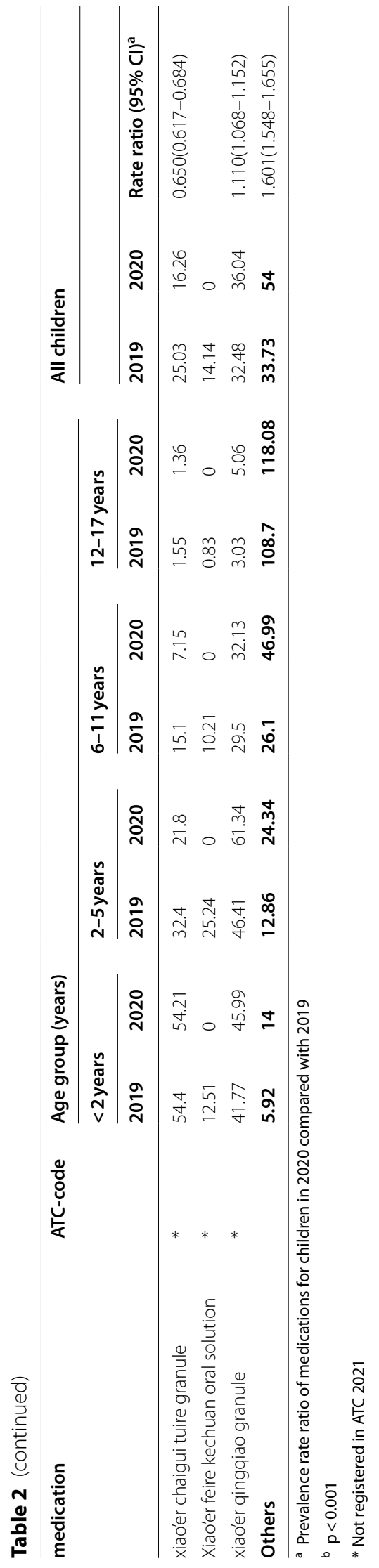




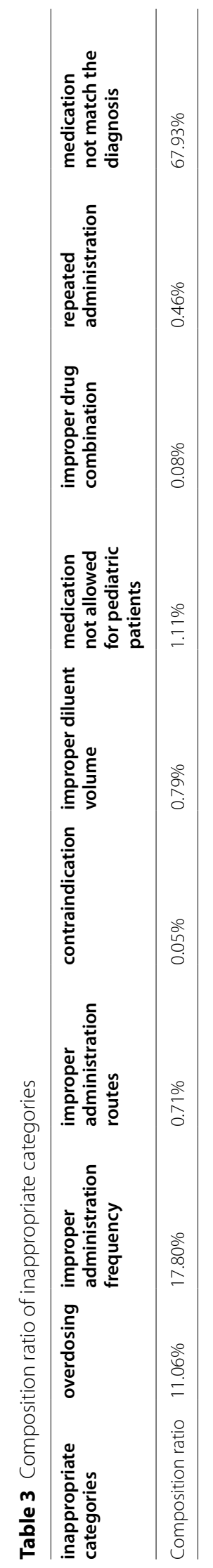




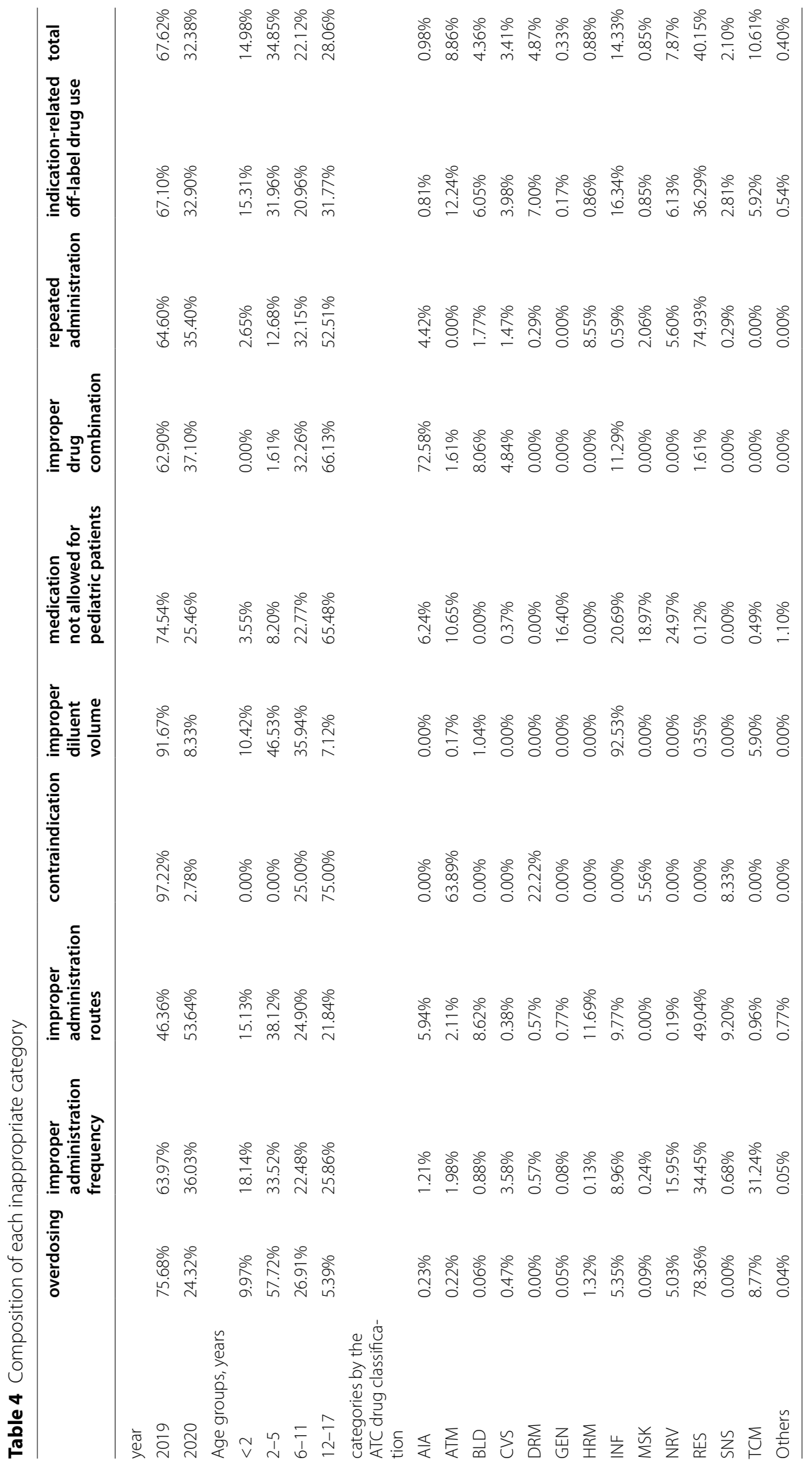




\section{Discussion}

This paper provides an overview of outpatient prescriptions and inappropriate medicinal use in the Chinese pediatric population. It assesses changes in prescription rates and prevalence of inappropriate medicinal use between 2019 and 2020. The study also identifies significant changes in the use of medicines before and after the outbreak of novel coronavirus pneumonia (COVID-19).

As an effect of COVID-19, the number of outpatient visits in Chinese general hospitals had decreased significantly [17]. For example, taking the author's hospital, the number of pediatric outpatients in 2020 was just $70.46 \%$ of that in 2019. To avoid gatherings and infection, pediatric patients tried to minimize the number of hospital visits, and doctors reduced the use of injected formulations. For the same purpose, doctors were more likely to prescribe more extended courses of medication to patients with chronic diseases to reduce the number of visits, so the spending on drugs per visit increased by $25.14 \%$ in this study.

Respiratory diseases are the most common disease in pediatrics [18-20]. This study found that $42.87 \%$ $(138,054 / 322000)$ of children who were prescribed medicines were diagnosed with respiratory diseases. Respiratory medicines, anti-infectives, and TCMs were most commonly used for respiratory diseases in children,and the number and prevalence rate of inappropriate use were also the highest. So the children who have respiratory diseases may have higher risks of inappropriate prescriptions, and doctors should be more cautious when prescribing drugs to these children.

The respiratory medicines were most widely used, and they appeared to be most inappropriately used. Budesonide was associated with severe off-label use related to indication, which accounted for $19.91 \%$ of all the improper medication use that related to indication.69.82\% (14,604/20916) budesonide was used either for pneumonia, tracheobronchitis, or upper respiratory trace infection. However, no evidence can be present from current research,that inhaled corticosteroids are effective in the treatment of cough due to acute tracheobronchitis and upper respiratory tract infection. Thus, it was not recommended to be used for routine respiratory tract infections $[20,21]$. Ambroxol was the second commonly used respiratory medicine, and the majority of ambroxol used was in injection form. In contrast, the use of oral preparations began just after March 2020 but was used only for 21 children all over the year. Ambroxol hydrochloride injection was used 18,598 times in 2019 and 2020 , and the prevalence reduced by $46.68 \%$ in 2020 than in 2019, with the decrease in the use of all injected formulations. In all the ambroxol hydrochloride injections used, $27.54 \%(5122 / 18598)$ were identified to have been overdosed. The overdosing of ambroxol hydrochloride injection accounted for $63.15 \%$ (5122/8111) of overdosing use of all the medicines. Several multi-center surveys on ambroxol hydrochloride injection in China indicated that the off-label drug use, especial off-label administration route, and off-label dosage were common; the incidence was $40-48 \%$ and $36-45 \%$, respectively [22, 23]. In the author's hospital, the off-label administration route of ambroxol was infrequent, and overdosing was lesser than in the hospitals above. Great attention needs to be paid, and more prospective trials are required to identify the effectiveness, safety, and necessity of off-label drug use of respiratory medicines. We believe that if the appropriate use of respiratory medications is increased, the rate of inappropriate prescriptions would be significantly reduced.

Since 2012, China has formally implemented a series of decrees for the clinical use of antibacterial agents [24]. However, the inappropriate use of antibiotics for respiratory tract conditions and viral infectious diseases still existed in pediatric outpatients. Third-generation cephalosporins and second-generation cephalosporins were the most commonly prescribed antibiotic classes, which accounted for $72.80 \%(71,283 / 97925)$ of all antibiotics used. However, these third-generation cephalosporins and second-generation cephalosporins were all WHO watch-group antibiotics and were not indicated to be prescribed for mild respiratory tract infections and viral infections [25]. The prevalence of improper administration frequency, overdosing, and improper diluent volume for injection in antibiotics was lower than $1 \%$ due to the strict antibiotic supervision by hospital management. It can be seen that the use of antibiotics under supervision has fetched some results, but more attention should be paid to the selection of antibiotics.

TCMs were widely used in children. Long-term clinical practice has confirmed that TCMs are safe and effective in treating acute upper respiratory tract infections in children [26]. In the case of COVID-19, the advantages of TCMs in treating respiratory diseases have been proved again $[27,28]$. In this study, the top 7 TCMs were all used for respiratory infections, in which three were pediatric drugs, whose dosage form and specifications were suitable for pediatric use. The other four TCMs were not pediatric drugs and had no specified doses for children. Doctors could only determine the dosage or administration frequency for these medicines according to their experience. This was also the main problem in the application of TCMs in children.

Dexamethasone was widely used in children, and $16.31 \%(22,510 / 138054)$ pediatric outpatients with respiratory diseases such as pneumonia, tracheobronchitis, or upper respiratory tract infections, were prescribed 
dexamethasone injection. The glucocorticoids were not recommended for routine use of respiratory diseases in various treatment guidelines in China. They can only be used to relieve symptoms when the patient has wheezing, breathing difficulties, and is at risk of suffocation $[18,29]$. Due to the limited information provided by the prescription, it was difficult to retrospectively and accurately determine whether each patient had the indication for the use of dexamethasone. Still, from the high use rate of dexamethasone, it could be inferred that a large number of dexamethasone was used inappropriately. Since the outbreak of COVID-19, the Department of Pharmacy and the Medical Service at the author's hospital organized a series of training on pneumonia and other respiratory diseases, including the rational use of glucocorticoids in respiratory diseases. The utilization of dexamethasone was reduced by $45.42 \%$ in 2020 compared to 2019 in this study, which could indicate that the use of glucocorticoids became more rationally in pediatric outpatients. However, the inappropriate use of glucocorticoids still exists, which requires more attention.

This study observed that only for $1.1 \%(3628 / 322000)$ pediatric outpatients, the cost of drug was covered by basic medical insurance, reflecting that the proportion of medical expenses paid by medical insurance for pediatric outpatients is very low. China has established a basic medical insurance system, covering more than $95 \%$ of the population in 2020 . However, at present, the basic medical insurance for children mainly focuses on inpatient and serious illness, but most children who fall ill occur in the outpatient department. On the other hand, the medicines out of the medical insurance catalog need to be paid on their own. Commercial health insurance for Children in China is still at the initial stage, and the coverage rate is very low $[2,30,31]$. So it was suggested that optimizing the design of basic medical insurance policies and formulating more favorable policies for children is quite necessary.

\section{Conclusion}

In general, compared to 2019, inappropriate drug use in pediatric outpatients in 2020 had significantly improved in hospital but is still common, especially in off-label use related to indication, doge, and administration frequency. In response to these situations, the Department of Pharmacy and the Medical Service at the author's hospital has adopted a series of interventions, such as specialized training for doctors, essential drug monitoring, and a list of off-label drug use, etc. In this process, the clinical rational drug use monitoring system plays a vital role in data monitoring, analysis, inappropriate prescription interception, etc. Since 2021, the clinical rational drug use monitoring system has started to intercept the inappropriate prescriptions of essential monitoring of drugs, which will significantly improve children's drug use's rationality, safety, and effectiveness.

\section{Limitations}

This study only involved one tertiary academic hospital, a teaching hospital, that can reflect the highest medical level in this province. Although it was strongly suggested by experts in China to establish the regional prescription circulation network between medical institutions and uniform prescription review and evaluation, there is still no successful case nationwide. Therefore, there is no multi-center data and a homogeneous prescription evaluation system among medical institutions currently.

The hospital did not implement electronic medical records before 2021, and doctors could only write medical records on paper. If the patient did not purchase the medicine prescribed by the doctor in the hospital, the information of the prescription could not be retained and could not be involved in this study.

\begin{abstract}
Authors' contributions
Cui Jing conducted statistical analysis and drafted the manuscript. Zhao Lei, Liu Xianghong and Liu Mengyujie participated in the statistical analysis and the writing of the manuscript. Zhong Lihong conceived the research, participated in the research design and coordination, and provided suggestions on the writing of the manuscript. All authors read and approved the final manuscript.
\end{abstract}

\section{Funding}

No funding was received for conducting this study.

Availability of data and materials Not applicable.

\section{Declarations}

\section{Ethics approval and consent to participate}

Ethical approval was granted by the Medical Ethics Committee of Qilu Hospital of Shandong University (Approval number KYLL-202011-136). All methods were carried out in accordance with relevant guidelines and regulations, and the Medical Ethics Committee of Qilu Hospital of Shandong University granted that informed consent could be waived.

Consent for publication

All authors consent for publication.

\section{Competing interests}

The authors declare that they have no competing interests.

Received: 25 June 2021 Accepted: 15 November 2021

Published online: 25 November 2021

\section{References}

1. National Bureau of Statistics. Main Data Bulletin of the Seventh National Population Census. 2010 .http://www.stats.gov.cn/tjsj/zxfb/202105/t2021 0510_1817181.html. Accessed 3 June 2021. 
2. Wang $X \mathrm{~L}$, Cao LJ, Liang YG. Establishing risk management system and insurance system for pediatric drugs in China. China Food Drug Administration Magazine. 2021;1:28-34

3. Xu XW, Wang YM, Li DK. Exploration of rational drug use in pediatrics. Chin J Clin Pharm. 2001:10(2):125-8.

4. Mehndiratta S. Strategies to reduce medication errors in pediatric ambulatory settings. J Postgrad Med. 2012;58(1):47.

5. Berthe-Aucejo A, Nguyen PKH, Angoulvant F, Bellettre X, Albaret P, Weil $T$, et al. Retrospective study of irrational prescribing in French paediatric hospital: prevalence of inappropriate prescription detected by pediatrics: omission of prescription and inappropriate prescription (POPI) in the emergency unit and in the ambulatory setting. BMJ Open. 2019;9(3):e019186.

6. Carvalho PR, Carvalho CG, Alievi PT, Martinbiancho J, Trotta EA. Prescription of drugs not appropriate for children in a pediatric intensive care unit. J Pediatr. 2003;79(5):397-402.

7. Landwehr C, Richardson J, Bint L, Parsons R, Sunderland B, Czarniak P. Cross-sectional survey of off-label and unlicensed prescribing for inpatients at a paediatric teaching hospital in Western Australia. PLoS One. 2019:14(1):e0210237.

8. Organization WH. Promoting safety of medicines for children: World Health Organization; 2007

9. Tomlin AM, Woods DJ, Reid JJ, Tilyard MW. Trends in prescription medicine use by older people in New Zealand 2010-2015: a national population-based study. N Z Med J. 2020;133(1513):61-72.

10. Christensen LD, Reilev M, Juul-Larsen HG, Jørgensen LM, Kaae S, Andersen $\mathrm{O}$, et al. Use of prescription drugs in the older adult population-a nationwide pharmacoepidemiological study. Eur J Clin Pharmacol. 2019;75(8):1125-33.

11. Forsman J, Taipale H, Masterman T, Tiihonen J, Tanskanen A. Comparison of dispensed medications and forensic-toxicological findings to assess pharmacotherapy in the Swedish population 2006 to 2013. Pharmacoepidemiol Drug Saf. 2018;27(10):1112-22.

12. National Health Commission of the People's Republic of China. Hospital Prescription Review Management Specification (Trial). http://www.gov. cn/gzdt/2010-03/04/content_1547080.htm. Accessed 3 June 2021.

13. International Council for Harmonisation of Technical Requirements for Pharmaceuticals for Human Use $(\mathrm{ICH})$. Clinical Investigation of Medicinal Products in the Pediatric Population E11 (R1). Adopted on 18 August 2017. https://database.ich.org/sites/default/files/E11_R1_Addendum.pdf. Accessed 3 June 2021

14. Zhang TT, Smith MA, Pat GC, Salomah S, Stuart MM, Bruce CC. Prescription drug dispensing profiles for one million children: a population-based analysis. Eur J Clin Pharmacol. 2013;69(3):581-8.

15. Wu F, Xie R, Xie SY. Analysis of the current situation of pediatric drugs use in the sample hosptial. Chin J Hosp Pharm. 2020;40(7):818-22.

16. WHO collaborating Centre for Drug Statistics Methodology. Anatomical Therapeutic Chem Code 2020. https://www.whocc.no/. Accessed 3 June 2021

17. Yao Z, Li XY, Zhang Y, Zuo Y, An FM, Wu YF, et al. Practice and thinking on the improvement of COVID-19 prevention and control work in general hospital outpatient management. Chinese Hospitals. 2021;25(2):88-90.

18. National health commission of the people's repablic of China. Guidelines for the diagnosis and treatment of community-acquired pneumonia in Children. 2019. http://www.nhc.gov.cn/yzygj/s7653/201902/bfa758ad6a dd48a599bc74b588a6e89a.shtml. Accessed 3 June 2021.

19. Li L, Liao X, Zhao J, Xie Y-M. Interpretation of chinese clinical guidelines for acute upper respiratory tract infection in children. Chin J Chin Materia Medica. 2017:42(8):1510-3.

20. Chinese Medical Association. Guideline for primary care of acute tracheobronchitis(2018). Chin J Gen Pract. 2019;4(18):314-7.

21. Pornsuriyasak $P$, Charoenpan $P$, Vongvivat $K$, Thakkinstian A. Inhaled corticosteroid for persistent cough following upper respiratory tract infection. Respirology. 2005;10(4):520-4

22. Zeng Y, Lin L, Lai S, Wen Y, Qian W, Chen L, et al. Multi-center survey on off-label drug use of Ambroxol hydrochloride injection. China Pharmacy. 2017;28(29):4060-5.

23. Fan QQ, Zhang B, Li DK, Mei D. Current clinical application of ambroxol hydrochloride injection and consideration on the rational drug use. Chin Pharm J. 2015;50(9):816-20.
24. Xiao Y, Li L. Legislation of clinical antibiotic use in China. Lancet Infect Dis. 2013;13(3):189-91.

25. Organization WH: Executive summary: the selection and use of essential medicines 2019: report of the 22nd WHO Expert Committee on the selection and use of essential medicines: WHO Headquarters, Geneva, 1-5 April 2019. In.: World Health Organization; 2019.

26. Rong P, Ma R, Liu Q-H, Yan H-H, Hu S-Y, Li X-M, et al. A commentary of literature research of traditional Chinese medicine for acute upper respiratory tract infection in children. Chin J Chin Materia Medica. 2017:42(8):1455-66

27. Li Y, Liu X, Guo L, Li J, Zhong D, Zhang Y, et al. Traditional Chinese herbal medicine for treating novel coronavirus (COVID-19) pneumonia: protocol for a systematic review and meta-analysis. Syst Rev. 2020;9:1-6.

28. Yang Y, Islam MS, Wang J, Li Y, Chen X. Traditional Chinese medicine in the treatment of patients infected with 2019-new coronavirus (SARS-CoV-2): a review and perspective. Int J Biol Sci. 2020;16(10):1708.

29. Chinese Medical Association. Guideline for primary care of acute upper respiratory tract infection(2018). Chin J Gen Pract, 2019(18)5:422-426.

30. Statistical Bulletin on the development of medical security in 2020. National Healthcare Security Administration. http://ybj.shandong.gov.cn/ art/2021/8/10/art_113607_10288884.html. Accessed 3 Jun 2021.

31. Xu N, Gu XF, Xiang GC. Review of Chinese Children's medical security policy. Health Economics Research. 2020;37(03):32-5.

\section{Publisher's Note}

Springer Nature remains neutral with regard to jurisdictional claims in published maps and institutional affiliations.
Ready to submit your research? Choose BMC and benefit from:

- fast, convenient online submission

- thorough peer review by experienced researchers in your field

- rapid publication on acceptance

- support for research data, including large and complex data types

- gold Open Access which fosters wider collaboration and increased citations

- maximum visibility for your research: over 100M website views per year

At BMC, research is always in progress.

Learn more biomedcentral.com/submissions 